\title{
NOTE ON DILWORTH'S DECOMPOSITION THEOREM FOR PARTIALLY ORDERED SETS
}

\author{
D. R. FULKERSON
}

Let $P$ be a finite partially ordered set with elements $1,2, \cdots, n$ and order relation denoted by " $>$." A chain in $P$ is a set of one or more elements $i_{1}, i_{2}, \cdots, i_{k}$ with $i_{1}>i_{2}>\cdots>i_{k}$. A decomposition of $P$ is a partition of $P$ into chains; a decomposition with the smallest number of chains is minimal. Two members $i, j$ of $P$ are unrelated if neither $i>j$ nor $j>i$. Dilworth [2, Theorem 1.1] has proved that the number of chains in a minimal decomposition of $P$ is equal to the maximal number of mutually unrelated elements of $P$.

Recently Dantzig and Hoffman [1] have formulated the problem of finding a minimal decomposition of $P$ as a transportation-type linear programming problem, and have shown that Dilworth's theorem follows from the duality theorem of linear inequality theory. Our aim here is to show that Dilworth's theorem can be deduced from the following theorem of König [4, p. 232]. Let $L$ be a linear graph with node set $N$ and suppose $N$ is partitioned into fixed subsets $N_{1}, N_{2}$. An $N_{1}, N_{2}$ cut $C$ of $L$ is a subset of $N$ having the property that every arc joining a node of $N_{1}$ to a node of $N_{2}$ has some node of $C$ as end point, and no proper subset of $C$ has this property. An $N_{1}, N_{2}$ join $J$ of $L$ is a set of $\operatorname{arcs}$ of $L$, each of which joins a node of $N_{1}$ to a node of $N_{2}$, and no two of which have a node in common. König's theorem, applied to the given $N_{1}, N_{2}$ partition of $L$, asserts that $\max _{J}|J|$ $=\min _{C}|C|$ (where $|S|$ denotes the number of elements in set $S$ ), the maximum being taken over all $N_{1}, N_{2}$ joins $J$, the minimum over all $N_{1}, N_{2}$ cuts $C$. A proof of this theorem has also been given by Egerváry [3].

We proceed to a deduction of Dilworth's theorem. Given the partially ordered set $P=\{1,2, \cdots, n\}$, let $L$ be the linear graph consisting of $2 n$ nodes, labeled $a_{1}, \cdots, a_{n}, b_{1}, \cdots, b_{n}$, and arcs defined from $P$ by the rule: If $i>j$, then $a_{i} b_{j}$ is an arc of $L$; these are all the arcs of $L$. Let $N_{1}=\left\{a_{1}, \cdots, a_{n}\right\}, N_{2}=\left\{b_{1}, \cdots, b_{n}\right\}$. Henceforth all joins and cuts are relative to $N_{1}, N_{2}$.

LEMMA 1. Corresponding to any join $J$ of $L$, there is a decomposition $D$ of $P$ with $|J|+|D|=n$.

Lemma 2. Corresponding to any cut $C$ of $L$, there is a set $U$ of mutually unrelated elements of $P$ with $|C|+|U|=n$.

Note that Dilworth's theorem follows from the lemmas and König's theorem. For let $\widehat{J}$ be a maximal join in $L, \widehat{C}$ a minimal cut,

Received by the editors June 28, 1955 and, in revised form, August 22, 1955. 
and let $\widehat{D}, \hat{O}$ be their respective correspondents in $P$. By König's theorem, $|\hat{J}|=|\widehat{C}|$; hence, by the lemmas, $|\hat{D}|=|\hat{O}|$. But $|U|$ $\leqq|D|$ for any $U$ and $D$, since two unrelated elements can not belong to the same chain. Hence $\max _{U}|U|=\min _{D}|D|$.

Proof of Lemma 1. Let $J=\left\{a_{i_{1}} b_{i_{2}}, a_{i_{3}} b_{i_{1}}, \cdots, a_{i_{2 k-1}} b_{i_{2 k}}\right\}$. Thus

$$
i_{1}>i_{2}, i_{3}>i_{4}, \cdots, i_{2 k-1}>i_{2 k}
$$

in $P$, and we may put these together to form chains in the obvious way. These chains will be disjunct, since $J$ is a join in $L$. By adding to these, as one-element chains, all indices from $1,2, \cdots, n$ which do not already occur, a decomposition $D$ of $P$ is obtained. If the length of the $i$ th chain in $D$ is $l_{i}$, then

$$
n=\sum_{i=1}^{|D|} l_{i}=\sum_{i=1}^{|D|}\left(l_{i}-1\right)+|D|=|J|+\mid D_{\mid} .
$$

Proof of Lemma 2. Let $C=\left\{a_{i_{1}}, \cdots, a_{i_{k}}, b_{j_{1}}, \cdots, b_{j_{m}}\right\}$. The elements of the set $I$ of indices $i_{1}, \cdots, i_{k}, j_{1}, \cdots, j_{m}$ are all distinct, for suppose $i_{1}=j_{1}$, say. Since $C$ is a cut, there is an $a_{r} \notin C$ with $a_{r} b_{j_{1}}$ an arc of $L$; similarly there is a $b_{s} \notin C$ with $a_{i_{1}} b_{s}$ an arc of $L$. Then, by the transitivity of the ordering and the assumption that $i_{1}=j_{1}$, it follows that $a_{r} b_{s}$ is an arc of $L$. This contradicts the fact that $C$ is a cut, and thus implies that the elements of $I$ are all distinct. Now let $U$ be the complement of the set $I$ in $1,2, \cdots, n$. Since $C$ is a cut, the elements of $U$ are mutually unrelated in $P$, and $n=|U|+|C|$.

It is true, conversely, that Dilworth's theorem implies König's theorem, as can be seen from Theorem 3.1 of [2] concerning set representatives. To prove this directly, make the linear graph $L$ with node partition $N_{1}=\left\{a_{1}, \cdots, a_{m}\right\}, N_{2}=\left\{b_{1}, \cdots, b_{n}\right\}$ into a partially ordered set $P$ by defining $a_{i}>b_{j}$ if $a_{i} b_{j}$ is an $\operatorname{arc}$ of $L$.

The desired implication now follows from the following two easily checked statements: (1) corresponding to any decomposition $D$ of $P$, there is a join $J$ of $L$ with $|D|+|J|=m+n$ (namely, let $J$ be the 2-element chains of $D$ ); (2) corresponding to any set of unrelated elements $U$ of $P$, there is a cut $C$ of $L$ with $|U|+|C| \leqq m+n$, for the complement of $U$ clearly contains a cut.

\section{REFERENCES}

1. G. B. Dantzig and A. Hoffman, On a theorem of Dilworth, "Contributions to linear inequalities and related topics," Annals of Mathematics Studies, no. 38.

2. R. P. Dilworth, $A$ decomposition theorem for partially ordered sets, Ann. of Math. (1) vol. 51 (1950) pp. 161-166.

3. E. Egerváry, Matrixok Combinatorius Tulajdonságaîrol, Matematikai es Fizikai Lapok vol. 38 (1931) pp. 16-28; (Trans. by H. Kuhn as Paper 4, Logistics Papers Issue No. 11, The George Washington University, 1954.)

4. König, Theorie der Graphen, New York, Chelsea, 1950.

RAND CORPORATION 